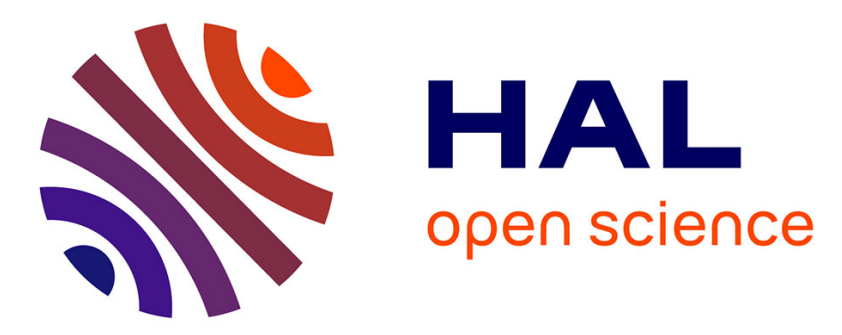

\title{
The Hyperfine Transition for the Definition of the Second
}

\author{
Elisa Felicitas Arias, Gérard Petit
}

\section{To cite this version:}

Elisa Felicitas Arias, Gérard Petit. The Hyperfine Transition for the Definition of the Second. Annalen der Physik, 2018, 531 (5), pp.1900068. 10.1002/andp.201900068 . hal-02183619

\section{HAL Id: hal-02183619 \\ https://hal.sorbonne-universite.fr/hal-02183619}

Submitted on 15 Jul 2019

HAL is a multi-disciplinary open access archive for the deposit and dissemination of scientific research documents, whether they are published or not. The documents may come from teaching and research institutions in France or abroad, or from public or private research centers.
L'archive ouverte pluridisciplinaire HAL, est destinée au dépôt et à la diffusion de documents scientifiques de niveau recherche, publiés ou non, émanant des établissements d'enseignement et de recherche français ou étrangers, des laboratoires publics ou privés. 


\title{
The Hyperfine Transition for the Definition of the Second
}

\author{
Elisa Felicitas Arias* and Gérard Petit
}

The unit of time of the International System of Units (SI), the "atomic second" was defined through a constant of physics in 1967. It is derived from the frequency of the hyperfine transition of the atom of cesium 133. From the astronomical definition of the second until today, the accuracy of the realization of the second has improved by eight orders of magnitude, with a rate that has increased since the development of the cesium frequency standards, to reach parts in $10^{16}$ for the best clocks today. In 2018, when the new SI was adopted, the time metrology community proved that a new generation of frequency standards operating in optical wavelengths has uncertainties at the level of $10^{-18}$, and challenge the implementation of high accurate frequency and time comparison techniques to decide on a revision of the definition of the second. Herein, the progress in the definition and realization of the second from astronomy until today is reviewed, an inventory of the present resources is assembled and a brief view for the future given.

in the hands of metrologists, with an unprecedented effort of international coordination and scientific cooperation.

The units of length and time had been the first to be defined through values of physical constants; in 1960 the metre was redefined in terms of the krypton radiation, and again in 1983 through the speed of light. In 1967 the second was redefined in terms of the frequency of the transition between the two hyperfine levels of the ground state of the 133 cesium atom. In 2018, the 26th CGPM anchored the SI by linking the other five base units of the international system to a set of defining constants drawn from the fundamental constants of physics and other constants of nature, from which the definitions of the base units are deduced.

\section{Introduction}

On 13 October 1967, government representatives at the 13th General Conference on Weights and Measures (CGPM) made the decision of redefining the SI unit of time as the second derived from the frequency of an atomic transition. This resolution was a dramatic turn-over that moved the activities on the realization and maintenance of the second from astronomy to physical sciences. While the relative accuracy of the current astronomical realization at the moment of the redefinition was of order $10^{-8}$, the cesium standard was estimated by the operators of such devices as four orders of magnitude more accurate.

Consequence of this change was the adoption of an atomic timescale as the world time reference; relevant international organizations and scientific unions working together concluded that International Atomic Time (TAI), although uniform, was not adapted to applications requiring a link to references on the rotating Earth, and defined Coordinated Universal Time (UTC), synchronized to rotational time better than $0.9 \mathrm{~s}$, still ruling today. The maintenance of the reference timescales has been since then

Dr. E. F. Arias

SYRTE, Observatoire de Paris

Université PSL

CNRS, Sorbonne Université

LNE, 61 avenue de l'Observatoire 75014, Paris, France

E-mail: felicitas.arias@obspm.fr

Dr. G. Petit

International Bureau of Weights and Measures

Pavillon de Breteuil, 92310 Sèvres, France

The ORCID identification number(s) for the author(s) of this article can be found under https://doi.org/10.1002/andp.201900068

DOI: 10.1002/andp.201900068
A new definition of the SI second could be expected in the next decade. Progress in the development of new frequency standards allows us to consider that clocks operating on optical transitions would be the candidates for redefining the second. They have demonstrated uncertainties of order $10^{-16}$ relative to the cesium, and a potential to reach standard uncertainties two orders better that the best microwave cesium standards. Still pending is the possibility of comparing these clocks over all distances with an uncertainty comparable to the clock's accuracy.

This paper presents the historical evolution of the definition of the SI second, focusing on its atomic definition and its consequences. The state-of-the-art on the development of cesium primary frequency standards (PFS) and their impact on timescale formation are also developed.

\section{Background}

Scientists do not intend to define time, but to establish the mechanisms to measure its flow. This had been, until the last half of the twentieth century, a task of astronomers. The celestial motions have been considered ever since as the natural phenomena providing the seemingly periodic events needed for the measurement of time. The man-made clocks were used as "time keepers," which frequency was adjusted by comparison with that coming from celestial motions. This procedure remained valid until the moment when clocks became more stable than the celestial motions used to steer their frequencies. The development of the first atomic clock in 1955 by Essen and Parry ${ }^{[1]}$ put time keeping in the hands of physicists, and more precisely metrologists. However, this transition was not without difficulty: time was no longer derived from a natural celestial phenomenon known by everyone, and in the perception of the epoch, coming 
from an "artificial" device operated in a laboratory made it poorly reliable.

In time keeping, the concepts of uniformity, time interval, and timescale are basic. Time cannot be measured without a timescale. Different timescales can coexist for various applications, but the unique timescale providing the time reference must be the most uniform; a time measurement is the measurement of a time interval. Prior to these concepts, the fundamental element is the unit.

To start, a natural, periodic phenomenon with an ideally unvarying frequency is chosen for the definition of the unit. Second, there is a need to identify a process and a device capable of reproducing the unit with some approximation, without interruption. This is the unitary scale interval. The timescale is finally generated with the adoption of an arbitrary origin.

The generated timescale will be uniform if 1) the unitary scale interval remains constant on a given time interval; a quantification of the capacity of reproducing the same unit interval over a lapse of time is the stability of the timescale; 2 ) the unitary scale interval agrees well with the definition of the unit; the level of agreement is the accuracy of the timescale. In fact, the concept of uniformity associated to a timescale is vague and it depends on the requested characteristics that are derived from the applications.

\section{Before Atomic Time}

\subsection{The Astronomical Definitions of the Second}

The first timescales were based on the repeatability of celestial motions where the underlying phenomenon responded to the requisite of being natural. As new clocks were being built and comparisons improved, instabilities of the natural phenomenon were revealed and could not be accounted for by improved modelling.

This was the case of Earth's rotation, reflected by the diurnal motion of the celestial bodies. It is necessary to note that a measurement of time is, in fact, the measurement of another quantity that can be assimilated or transformed to obtain time. Apparent solar time is defined as "the hour angle of the Sun," the $360^{\circ}$ of the diurnal path of the Sun correspond to $24 \mathrm{~h}$, so that $1 \mathrm{~h}$ is equivalent to $15^{\circ}$ of hour angle. The irregularities of the apparent solar time were already understood in the times of Ptolemy. The laws of planetary motions explained that the orbit of the Earth around the Sun was elliptical, and that it was tilted with respect to the plane defined by the Earth's rotation. A uniform time was then derived by correcting the effects of the ellipticity of the Earth's orbit and of its tilt with respect to the equator, under the name mean solar time. It represented the time of a fictitious Sun moving on the equator with a constant velocity equal to the mean annual velocity of the real Sun. This concept was associated to the local meridian. The nineteenth century brought the necessity of unifying the time, at least on national level due in part to the extension of the railways. The concept of "official" or "legal" time of a nation was born, and some countries defined as official hour that of an arbitrarily adopted meridian crossing their territories. The next step was to create the concept of a unique time, "universal" in the sense of being the same for everyone. This was possible

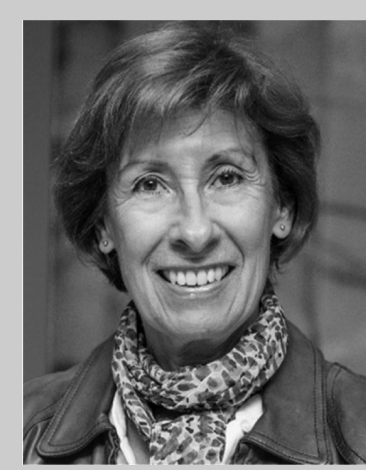

Felicitas Arias received the master's degree in astronomy from the University of La Plata (Argentina) and the Ph.D. in astrometry, celestial mechanics and geodesy from Paris Observatory. She was director of the Buenos Aires Naval Observatory and Professor at the University of La Plata. She was Director of the Time Department of the International Bureau of Weights and Measures (BIPM) from where she retired end of 2017. She collaborates with the LNE-SYRTE at Paris Observatory. Her fields of activity are the space and time references.

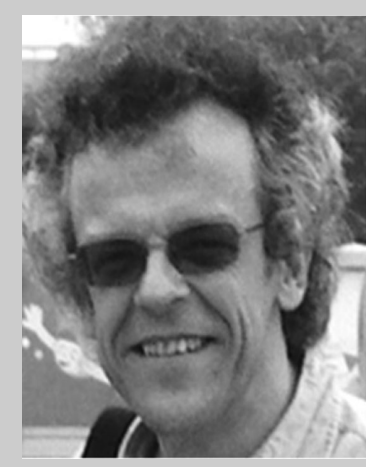

Gérard Petit graduated from Ecole Polytechnique, France, in 1979. He obtained a Ph.D. in astronomy from Paris Observatory in 1994. After working in the field of geodesy, he joined the International Bureau of Weights and Measures (BIPM) where he now is a Principal Research Physicist with the Time Department. His research interests concern all aspects of the elaboration and usage of timescales, especially time and frequency transfer techniques using GNSS, relativistic treatment for time and frequency applications and the use of frequency standards in generating International Atomic Time TAI.

with the adoption, in 1884, of a prime meridian, and a universal time, defined as the mean solar time corresponding to the prime meridian. The Earth's globe was divided in 24 time zones of $1 \mathrm{~h}$, the first centered on the Greenwich meridian, adopted as prime meridian. This was the first step toward international time coordination, when the countries adopted legal times in correspondence with the time zones.

The establishment of timescales had been since then in the domain of astronomy. The second was defined as the 1/86 400 part of the mean solar day. In 1948 the International Astronomical Union (IAU) recommended the use of Universal Time (UT). A determination of universal time implied astronomical observations to measure the sidereal time. Sidereal time is the hour angle of the equinox reckoned from the meridian (it has the denomination Greenwich sidereal time when it is referred to the Greenwich meridian). With the help of transit instruments, the instants of meridian transit of stars were detected, and the corrections to the reference clock were derived. Clocks had the role of "time keepers," and they were not at all associated to the phenomenon underlying the timescale. Clocks provided, 
through extrapolation, real time UT, and they produced time signals for time dissemination.

The first observational indication that the Earth's rotation rate was variable was made using high-grade pendulum clocks, and confirmed following the development of crystal clocks in the 1930s by Scheibe and Adelsberger of the PhysikalischTechnische Reichsanstalt (PTR) in Germany. ${ }^{[2]}$ The astronomers were thought to have found the solution to the problem of defining a uniform timescale with the adoption of Ephemeris Time (TE) in $1952^{[3]}$; a form of dynamical time, generally defined as the argument in the dynamical equations of the bodies of the Solar System, and related to the geometrical mean longitude of the Sun by Newcomb's theory. ${ }^{[4]}$ The practical realization of TE was not based on the Solar motion but rather on the lunar motion, since it was the most easily observable object in the solar system. It relied on the dynamical theory of the Moon and on observations, and any improvement in the theory would imply a redefinition of the timescale. The 11th CGPM in 1960 adopted the ephemeris second as the SI unit of time..$^{[5]}$ It was defined as a fraction of the tropical year 1900, thus in retrospective, and not susceptible of being reproduced, what was a clear drawback. The duration of the ephemeris second equals approximately the duration of a second of mean solar time averaged over the previous century. As it turned out, it was in fact shorter by $1.4 \times 10^{-8} \mathrm{~s}$ when averaging around year 1960.

TE was available in the form of a correction to UT with oneyear delay; it has never been used in civil time due to the fact of its late availability and because there was no form of dissemination. TE has been confined to astronomers, while UT remained the timescale for practical use.

It is worth noting that, in a certain manner, this was the kick-off of the international cooperation that today makes international time keeping a success: in the 1950s, the precision in the determination of time was a few milliseconds, and the delay to obtain definitive results was one year; in the twenty-first century we determine the international time reference with a few nanoseconds precision, with a delay of one month.

\subsection{The Dawn of Atomic Time, the First Cesium Standard}

Work at the US National Bureau of Standards (NBS, today National Institute of Standards and Technology, NIST) constituted the first steps toward the construction of atomic clocks. In 1937 Isidor Rabi invented the molecular beam magnetic resonance method, ${ }^{[6]}$ and observed the first atomic hyperfine structure transitions. ${ }^{[7]}$ In 1949 Lyons linked a resonating quartz crystal to an ammonia spectral line at a frequency of $24 \mathrm{GHz}$, with a relative accuracy of some parts in $10^{7}$. Norman Ramsey's work between 1950 and 1955 facilitated the achievement of the atomic clock. He developed a method ${ }^{[8]}$ where the atoms are observed successively along two electromagnetic fields in two cavities allowing observing very narrow resonances, and consequently obtaining a higher quality factor than those obtained with only one interaction (see also Section 8.1). In 1955 Louis Essen and Jack Parry from the UK National Physical Laboratory (NPL) achieved the first operational cesium atomic clock using Ramsey's method which was to become a standard of frequency.

\section{The Adoption of the Atomic Definition of the Second}

The second defined through the equations of motion of the Solar system in 1952 by the IAU, and ratified in 1960 by the CGPMthe ephemeris second-had a short life; it was not reproducible and the associated timescale, called ephemeris time, was used only by a small fraction of astronomers. It was defined as derived from the orbital motion of the Earth, but in practice computed from the dynamical theory of the Moon, the best observed object in the sky. It was realized with about one year delay under the form of corrections to Earth rotation time. Some years after its introduction, the astronomers discussed on the possibility of improving the ephemeris time by adopting a better theory and improved models. But the fact that atomic clocks already existed and were operated in a few laboratories, and that the realization of the unit of time through an atomic transition was possible in real time, reproducible, and more accurate than celestial motions, led to considering a dramatic change in the definition.

The adoption of the ephemeris second was certainly an unnecessary step toward the atomic second. The rational explanation is that it was not certain, at this epoch, that the cesium transition was the best choice and that all the corrections due to the perturbations of the atoms were considered; indeed, a small correction due to the black body radiation was applied much later. The possibility that atomic time differed from dynamical time was also evoked. In fact it can be considered that the need to explicitly use a relativistic formalism later clarified the relations between such different timescales. Less rational, but strongly anchored in society, was the feeling that time could be derived only from the motion of celestial bodies. Nevertheless, under the pressure of physicists, a value of the frequency for cesium standards was suggested for temporary use by the International Committee for Weights and Measures (CIPM) in1964.

\subsection{The Continuity of the Successive Definitions}

One basic rule of metrology is the continuity of the numerical value of the unit when the physical definition is changed. As expressed by Leschiutta, ${ }^{[9]}$ this practice guarantees that by changing the unit no bias is introduced in series of measurements covering different unit definitions.

There is no indication of any formal adoption of the second as the fraction $1 / 86400$ of the mean solar day as the unit of time, as it was assumed for centuries. The twentieth century put light on two characteristics of the Earth's dynamics: the motion of the rotation axis within the Earth, known as polar motion, and the irregularities of the Earth rotation. Later on, improvements were introduced to account for these effects. To avoid variations of order $10^{-7}$ to $10^{-8}$ in relative value, the astronomers suggested a new definition of the second based on the orbital motion of the Earth. The ephemeris second was defined by the IAU as the fraction 1/31 556925.9747 of the tropical year starting on 1900 January 0 at $12 \mathrm{~h}$ ephemeris time. This relation was obtained from the expression of the Sun's mean longitude in the theory of the Sun of Newcomb ${ }^{[4]}$

$L=279^{\circ} 41^{\prime} 48.04^{\prime \prime}+129602768.13^{\prime \prime} T+1.089^{\prime \prime} T^{2}$ 
where $T$ is the time reckoned in Julian centuries of 36525 days.

In fact, the ephemeris second was shorter than the mean solar second by $1.4 \times 10^{-8}$ at the time of its adoption in 1960, thus breaking somehow the principle of continuity between successively defined units. The atomic second, adopted years later, was linked to the ephemeris second, and in consequence maintained the difference with the mean solar second.

The next change in the definition of the time unit involved also a change in the paradigm and introduced the complexity of finding the equivalence between two seconds with completely different physical definitions. It consisted in comparing the frequency based on astronomical observations to the frequency of an atomic transition; however, the two experiments were in two remote locations and could not be directly compared. The solution was to independently relate two clocks to the two phenomena, and to compare their output frequencies. Simple in principle, but with some problems to overcome: to have stable clocks, able to maintain a constant rate over the interval of the astronomical observations; to link the atomic transition to a frequency standard; to make time and frequency comparisons between the two remote clocks using the methods available in the 1960s.

The link of a clock to astronomical observations was achieved with an instrument and method designed at the US Naval Observatory (USNO) in Washington by William Markowitz and R. Glenn Hall. ${ }^{[10]}$ The "Moon camera" designed by Markowitz was a photographic instrument used to observe occultations of stars by the Moon, reckoning the times of the observations.

The clocks used in the experience were the state-of-the-art clocks in the early 1960s, piezoelectric clocks, kept in controlled environments. The measurements to link the Essen cesium frequency standard to the crystal clock took place at the NPL, in the United Kingdom. Essen's clock was in discontinuous operation, relying on the piezoelectric standard as the flywheel during the non-operation intervals. The connection between the two clocks across the Atlantic Ocean was achieved using worldwide coordinated time signals. Several institutions and individuals contributed to the success of this task.

Markowitz and Hall, at the USNO determined the frequency of the cesium in terms of the ephemeris second; this calibration led to the value of the cesium frequency of $9192631770 \mathrm{~Hz}$, with an uncertainty of $20 \mathrm{~Hz}$. This value was adopted by the 13th CGPM in 1967 for the definition of the SI second. ${ }^{[1]}$ We should note that the value obtained by Essen and Parry several years before was remarkably close (9 $192631830 \mathrm{~Hz}$ ), differing only by $7 \times 10^{-9} \cdot[12]$

Figure 1 shows the relative accuracy of the successive realizations of the second, from some $10^{-8}$ (rotational second) to some $10^{-16}$ for the best present atomic standards.

\section{The International Coordination}

More than any other physical quantity, decisions on time and time unit have been made from discussions among many scientific organizations. Since the signature of the Metre Convention in 1875 , resolutions on the definition and realization of the units of measurement are a responsibility of the CGPM. In spite of this, the realization of the second has been until 1967 a matter of astronomers, thus requiring close interaction with the IAU.

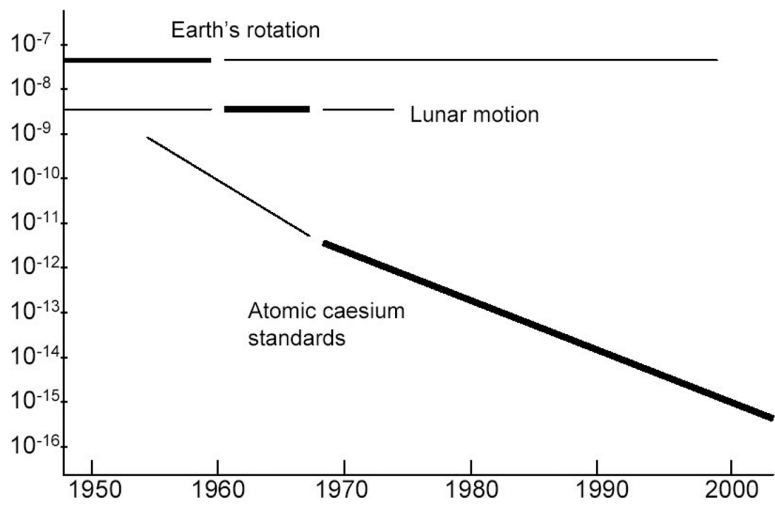

Figure 1. Evolution of the relative accuracy of the successive realizations of the second. The figure shows the relative fluctuations of the duration of the Universal Time second (Earth's rotation), of the duration of the second of Ephemeris Time, as realized by lunar observations, and as realized by $\mathrm{Cs}$ frequency standards. A thicker line represents the periods of official definition of the second. Reproduced with permission. ${ }^{[13]}$ Copyright 2005, IOP.

A coordinated effort was done in 1912, with the creation of the Bureau international de l'heure (BIH) based at Paris Observatory. The mission of the BIH was to provide a form of universal time, and to give the discrepancy between this reference time and that emitted by time signals. In the 1940s these discrepancies were of the order tenths of a second. The period 1950-1967 was rich in discussions on the achievement of uniform time and a convenient associated unit. Personalities of the metrology and astronomy communities shared discussions at the IAU Commission on Time and at the Consultative Committee for the Definition of the Second (CCDS) created by the CIPM. In 1952, the IAU Commission on Time ${ }^{[14]}$ presented the problem of the coexistence of two time systems, the uniform ephemeris time, and that derived from the irregular rotation of the Earth; for the accurate day-to-day control of their differences quartz clocks were not stable enough, but it was already recognized that a possible solution would be the atomic or molecular clocks, still under development at that time. In 1955, when the time services and observatories operated quartz crystal clocks, the decision of the adoption of the ephemeris second as the unit of time was made at the IAU with the accord of Essen and Markowitz. ${ }^{[15]}$ Therefore, the CIPM in 1956, ${ }^{[16]}$ followed the decision of the IAU and proposed a new definition of the second as a fraction of the tropical year of 1900 . This decision was ratified by the 11th CGPM in $1960 .{ }^{[4]}$

The progress in the development of cesium atomic standards, their operation in some metrology institutes, and the understanding that they could realize the second with unprecedented accuracy moved the CCDS to recommend in 1967 that the unit of time of the SI is that realized with the isotope 133 of the cesium atom, and that the second defined by the CGPM in 1956 be called "ephemeris second." This recommendation was welcomed by the IAU. The CCDS recommended also that the CIPM should promote a meeting with the BIH, the IAU, the International Union of Geophysics and Geodesy (IUGG), the International Union of Radio Sciences (URSI) and the International Consultative Committee of Radiocommunications (CCIR) of the International Telecommunications Union (ITU) to study the problems issuing from the adoption of the new unit of time. These organizations gathered all scientific activities related 
to Earth, space, and time references, and represented the most important communities of users. Such coordination had never existed for the determination of ephemeris time.

A consequence of the new definition was the construction of an atomic timescale based on the atomic second. From the interaction of representatives of the organizations convocated by the CIPM, two timescales were created; a uniform timescale based on the atomic second named International Atomic Time, which should be the basis of the construction of the international time reference, Coordinated Universal Time. TAI was constructed at the $\mathrm{BIH}$ with the cooperation of the institutes realizing local atomic timescales; since the ITU established the rules for the emission of standard frequencies and time signals, it was at its CCIR that the mechanism for obtaining UTC through the insertion of a leap second was described. ${ }^{[17]}$ The BIH was also responsible for predicting and announcing the dates of insertion of leap seconds.

The BIH suffered a transformation consequence of the adoption of space techniques for the monitoring of the irregularities of the Earth's rotation. In 1988 it was officially closed, and the International Earth's Rotation and Reference Systems Service (IERS) took the responsibility for maintaining the terrestrial and celestial reference systems and the parameters of orientation of the Earth. The maintenance of the timescales TAI and UTC was transferred to the International Bureau of Weights and Measures (BIPM), putting under the umbrella of the Metre Convention all the activities relating to the time references. Only the decision on the date of insertion of leap seconds in UTC remains a task of the IERS.

In 1992 the CCIR became the Radiocommunication Sector of the ITU (ITU-R), with the responsibility of developing standards for radiocommunication systems. In 1997 the CCDS was renamed Consultative Committee for Time and Frequency (CCTF), to better reflect its enlarged scope of activity, which included not only issues related to the definition of the second, but also progress on clocks, time and frequency comparisons and timescales.

The joint work of the international and scientific organizations made the international time system a success.

\section{The Atomic Standards}

We should understand the difference between the atomic standards developed by some laboratories with the aim of realizing the atomic second and those fabricated in industries to be commercialized for different applications. The first group involves the primary frequency standards that realize the second with ultimate accuracy, see Section 8 for a detailed description of the primary frequency standards, their impact and applications. In the second group the industrial standards have practical properties, for example, small size, good cost-to-value ratio, or easy operation. One of their more demanding applications is for timescale realization at metrology laboratories.

Strictly speaking, in time metrology a clock is a device on continuous operation. Although some primary cesium standards (magnetic beams and fountains) operate without interruption since many years, atomic clocks developed industrially are used to construct atomic timescales. Clocks of this kind operate in all laboratories maintaining local representations of the world time reference UTC, contribute to the generation of the internal timescales essential to the operations of global navigation satellite systems, and are used in other non-metrological applications. They guarantee the stability of a timescale at different time intervals and are generally accurate to the level of $10^{-12}$ or $10^{-13}$ at best. In all cases, the timescales generated by them are steered to a more accurate reference (a primary standard or another timescale, as is explained in Section 8.2.2).

Among cesium industrial clocks most have similar design as the Essen standard, the cesium beam clock with magnetic deflection. The first commercial cesium clocks were constructed in Switzerland (Ebauches), and the United States (HewlettPackard). Since 1992 various fabricants provided successively the model 5071A, operated in almost all the time and frequency metrology laboratories for its good stability, better than $1 \times 10^{-14}$ at intervals ${ }^{[18]}$ of five days or more, and accuracy of about five parts in $10^{13}$.

Another atomic standard is the hydrogen maser (Microwave Amplification by Stimulated Emission of Radiation) clock. The atom is stimulated to emit at the frequency of the ground-state hyperfine splitting $(1.4 \mathrm{GHz})$ of atomic hydrogen. Clearly, they are not accurate since they do not operate with cesium, and hence, not according to the definition of the second, but they are the most stable in the market, about 100 times more stable than a cesium clock up to seven days of operation. About $25 \%$ of the atomic clocks in laboratories contributing to the computation of international atomic time are hydrogen masers; due to their excellent short-term stability they improve the timescales locally realized and link the primary frequency standards to international atomic time.

\section{A Consequence of the Atomic Definition of the Second: The Adoption of TAI and UTC}

In the 1960 s the BIH maintained universal time from astronomical determinations of time and latitude in a group of observatories in different nations. This time was affected by the motion of the terrestrial axis of the Earth and by the variable velocity of rotation of the Earth. A form of Universal Time denominated UT1 is derived eliminating the effects of polar motion; it is proportional to the rotation angle of the Earth in space. UT1 is affected by a secular deceleration and decade fluctuations. If seasonal fluctuations are eliminated from UT1, we obtain UT2.

Experiments with atomic clocks already existed at the moment of the definition of ephemeris time; it can be considered as a "transition time" between the astronomical and the physical timescales. Laboratory cesium standards were constructed in some laboratories and short time later commercial cesium standards became available. This gave rise to independent atomic times TA(k) in the United States of America at the USNO and the NBS (today NIST), and in Europe at the Royal Greenwich Observatory (RGO, United Kingdom) and the Physikalisch-Technische Bundesanstalt (PTB Germany), and there rose two problems: comparing them at the level of performance of the standards, and averaging them to produce a mean atomic timescale more uniform and reliable than the individual ones. Atomic clock transportation contributed to improve the comparisons, allowing time 
transfer with an uncertainty of $1 \mu \mathrm{s}$. In 1968 the clock comparison had much evolved. The system of radionavigation LORAN-C (Long Range Navigation), the clock transportation, the frequencies broadcasted by VLF (Very Low Frequency), and the clock synchronization by television allowed time transfer with uncertainties of order 10 to $1 \mu \mathrm{s}$.

The BIH maintained an atomic timescale named TA(BIH) based on local independent atomic scales maintained in laboratories in Europe and the USA.

After the adoption of the atomic definition of the second in 1967, the next step was to define a timescale based on the new unit. The acceptance of a timescale of atomic nature was not straightforward. Some of the major objections concerned the fact that being an integrated timescale, constructed from a succession of seconds, the uncertainties do not decrease with the improvement of observations, but they are also integrated; making a difference with the astronomical timescales.

In spite of the objections, atomic time was accepted. The adoption of the atomic timescale of the BIH was recommended successively by the different organizations: the IAU in 1967, the URSI in 1969 and the ITU-CCIR in 1970. The 14th CGPM in 1971, requested the CIPM to define and establish International Atomic Time with the acronym TAI, recognizing that the BIH had already constructed an atomic timescale of the desired quality. ${ }^{[19]}$

TAI has never been disseminated directly; UTC, approximating UT1, has been adopted as the international time reference since 1972, with the endorsement of the 15th CGPM in 1975. ${ }^{[20]}$ Its definition almost 50 years ago responded to a need in real time for some specific applications including astronomical navigation, geodesy, telescope settings, space navigation, satellite tracking, etc. The definition of UTC derives from the tolerance for the time offset [UT1-UTC]. Since 1972, UTC has differed from TAI by an integer number of seconds, changed when necessary by insertion of a leap second to maintain $|U T 1-U T C|<0.9 \mathrm{~s}$. Although this system works well, it seems that no application demands this coarse access to UT1 by means of UTC today; in addition, leap seconds become increasingly cumbersome, introducing an ambiguity in dating events when they occur. With the progress of communications, other means to provide UT1 in real time can be conceived and the future of UTC is being discussed.

UTC is the international reference for time coordination. UTC is a "paper timescale," accessible after the fact through its offsets with respect to its local realizations, indicated UTC $(k)$. A number of institutes distributed worldwide (85 in January 2019) maintain such atomic timescales that represent local approximations of UTC. These institutes operate in total about 450 atomic clocks (industrial cesiums and hydrogen masers), and about a dozen primary and secondary frequency standards. The degree of approximation of these timescales with respect to the reference is established monthly by the BIPM, together with their respective uncertainties. ${ }^{[21]}$ The best realizations approximate UTC to better than 2.5 ns RMS over many years. Their frequencies are steered to a reference (UTC or a primary frequency standard) to improve their accuracies. The UTC $(\mathrm{k})$ are practical timescales, produced and disseminated in real time. Their dissemination is possible by different means, either the classical emissions of time signals and standard frequencies following the rules of the ITU-R, by telephone, or more in the rhythm of today, through the internet. The UTC $(k)$ are the basis of most national legal times.
TAI and UTC have numerous applications in time synchronization at all levels of precision; from the second needed by the general public, to the nanoseconds required in the most demanding applications. TAI is the basis of realization of timescales used in dynamics, for modelling the motions of artificial and natural celestial bodies, with applications in the exploration of the universe, tests of theories, geodesy, geophysics, studies of the environment. In all these applications, relativistic effects are important.

Although TAI and UTC have represented the standard frequency and time references in the last five decades, their formal definition has been adopted only in 2018 by the 26th CGPM, ${ }^{[22]}$ repairing an oblivion that brought confusion on the sharing of responsibilities between the BIPM, the IERS, and the ITU on the definition, realization, and dissemination of UTC.

\section{Primary Frequency Standards}

Resolution 1 of the 13th CGPM states that "The second is the duration of 9192631770 periods of the radiation corresponding to the transition between the two hyperfine levels of the ground state of the cesium 133 atom". This statement refers, without saying, to "unperturbed" atoms, that is, those at rest, at zero magnetic and electric fields. We define PFS as those frequency standards that aim at exactly realizing the SI second using the transition in the definition and for which corrections with respect to all known systematic shifts have been applied to best knowledge. In addition, Secondary Frequency Standards (SFS) realize one of the transitions which have been recognized as secondary representations of the second by the CCTF, see the list at https://www.bipm.org/en/publications/mises-en-pratique/ standard-frequencies.html. Primary and secondary standards are collectively referred to as PSFS.

PFS provide accuracy to the timescales generated by the BIPM: TAI which is basis of the world's time reference UTC, and the post-processed timescale TT(BIPM). ${ }^{[23]}$ This presentation focuses on primary standards but, in addition to PFS, secondary frequency standards have also effectively been contributing to the accuracy estimation of TAI and to the generation of TT(BIPM) since 2012 .

In the following we first review the two main generations of PFS that can be clearly distinguished, that is, the first generation of atomic beam standards and the second generation of atomic fountains. We then emphasize the impact of atomic fountains on timescales through their role in TAI steering and their use in the generation of the local timescale UTC $(\mathrm{k})$ of some laboratories.

\subsection{Two Generations of Primary Frequency Standards}

Progresses in PFS have been continuous since the 1967 definition of the second. The first generation of thermal atomic beam devices progressed by about two orders of magnitude until reaching the relative accuracy level of $1 \times 10^{-14}$ around year 2000, and some of these standards are still operating today. This improvement prompted a clarification to the 1967 definition by the CIPM which, at its 1997 meeting affirmed that the definition 
refers to a cesium atom at rest at a temperature of $0 \mathrm{~K}$. This note was intended to make it clear that the definition of the SI second is based on a cesium atom unperturbed by black body radiation, that is, in an environment whose thermodynamic temperature is $0 \mathrm{~K}$. Indeed this effect, causing a frequency shift of order $1 \times 10^{-14}$, could not be neglected anymore and the frequencies of all PFS should therefore be corrected for the shift due to ambient radiation, as stated at the meeting of the CCDS in 1996.

The second generation of devices, the Cs fountains, started at the end of the $1990 \mathrm{~s}$ with relative accuracy in the $10^{-15}$ range and has now reached relative accuracy of $1-2 \times 10^{-16}$ in the best cases.

Even though it is not the purpose of this paper to enter into technical details we may consider one parameter that is fundamental in atomic clocks, the quality factor, that is, the ratio $Q \approx v / \Delta v$ between the frequency of the transition and the linewidth of the resonance. The instability of the standard is proportional to $1 / Q$ and the instability also conditions, to a large level, the ability to determine the systematic shift thus the accuracy of the standard. The two generations of Cs standards are, by principle, separated by about two orders of magnitude in quality factor thus also by two orders of magnitude in overall performance, as reviewed below.

\subsubsection{Cs Beam Standards}

The first generation of atomic Cs clocks started with the work of Essen and Parry ${ }^{[1]}$ and is based on the interaction of a beam of Cs atoms with a synthesized microwave field in a microwave cavity so that the frequency of the field may be tuned to the frequency of the $9.192631770 \mathrm{GHz}$ atomic transition. The scheme takes advantage of the separated oscillatory field approach proposed by Ramsey to improve the stability of the clock by increasing the interaction time thus the quality factor. The literature describing the progresses of these clocks is vast and we do not need to add a detailed presentation here, we instead direct interested readers to, for example, the special issue of Metrologia 42 for the 50th anniversary of the Essen and Parry publication, particularly the papers by Vanier and Audoin ${ }^{[24]}$ and Ramsey. ${ }^{[25]}$

An interesting development in the first generation was the development of optical pumping using lasers to pump atoms in the desired quantum state and minimize the frequency pulling by neighboring transitions. Such devices were operated in the late 1990 s eventually providing a relative accuracy somewhat below $1 \times 10^{-14}$. Nevertheless, in the end, the relatively high speed of the thermal atoms $\left(\approx 100 \mathrm{~m} \mathrm{~s}^{-1}\right)$ fundamentally limits the stability and accuracy of the Cs beam devices to somewhere around $1 \times 10^{-14}$ or slightly below.

Such standards have been developed in several metrology institutes. Here we mention those that were used at some point for TAI since 1972:

- at the NIST (Boulder, USA), then named the NBS, with the series NBS-4 to NBS-6 followed by NIST-7 (with optical pumping) in the 1990.

- at the PTB (Braunschweig, Germany), where the standards CS1 and CS2 are still operated today.
- at the Paris Observatory (now LNE-SYRTE), where the JPO (with optical pumping) was operated until 2010.

- at the NRC (Ottawa, Canada), the RRL (Tokyo, Japan, now NICT) where NICT-O1 (with optical pumping) was operated until 2006, the NRLM (Ibaraki, Japan), the KSRI (now KRISS, Daejeon, Korea), the VNIIFTRI (Moscow, Russia),

See Section 8.2 for more details on the use of these standards for TAI.

\subsubsection{Cs Fountains}

The original concept of a cesium fountain was introduced in the 1950 s by Zacharias, ${ }^{[26,27]}$ in the broad form of a vertical Cs beam clock with one Ramsey interaction zone that would be traversed by the atoms twice, first upward by the traveling atoms and then downward after the atoms fall back under gravity. However, this concept did not work for lack of usable slow atoms from an atomic beam and was reactivated only when the advent of laser cooling technique allowed a better approach to the problem. Methods of laser cooling and trapping atoms allowed capturing a large number (tens of millions) of atoms at low thermal velocity (of the order of $1 \mathrm{~cm} \mathrm{~s}^{-1}$ ) so that they stay together for a significant time and can subsequently be launched against gravity using laser light. These techniques, for which the 1997 Nobel Prize in Physics was awarded to Claude Cohen-Tannoudji, Steven Chu, and William Daniel Phillips, were fundamental in making the atomic fountain concept successful.

Again it is out of the scope of this paper to describe in any details the numerous technical developments that allowed fountains to work in the first place, and then to drive their uncertainty budget from the few $10^{-15}$ to the low $10^{-16}$ in about 15 years. More details may be found, for example, in the work by Wynands and Weyers. ${ }^{[28]}$

Let us just mention a few items:

- The main limitations to Cs fountains, around the $1 \times 10^{-16}$ level, seem to be due to the blackbody frequency shift, the shift due to collisions, and also the cavity phase shift.

- Some groups (NIST, INRIM) have tried to minimize the effect of the blackbody frequency shift, which is proportional to $T^{4}$, by building a cryogenic ( $T=77 \mathrm{~K}$ ) vacuum structure that includes the microwave cavities and flight tube above them. ${ }^{[29,30]}$

- The collisional shift would have been less of a problem in, for example, $\mathrm{Rb}$ fountains where it is nearly two orders of magnitude lower than in Cs.

- A limitation in the stability of any atomic clock with pulsed operation, like most fountains, is the Dick effect. ${ }^{[31]}$ Options to minimize it are to use an extremely good local oscillator, to shorten the dead time of the pulsed operation, or to build a continuous-beam fountain. The last option has been put into operational use by METAS in its FOCs2. ${ }^{[32]}$

\subsection{Impact of Fountains on Timescales}

Since they became operational at the end of the twentieth century, atomic fountains have improved and grown in all aspects. 
Table 1. Evaluation of the frequency of TAl by primary standards over 1975 and previous years (Data taken from Table 23 of ref. [33]).

\begin{tabular}{|c|c|c|c|c|c|}
\hline LAB. & STANDARD & $\begin{array}{l}\text { CALIBRATION INTERVAL } \\
\text { MJD }\end{array}$ & $\begin{array}{l}\text { NORMALIZED FREQ. DIF. OF } \\
\text { TAI - STAND. IN } 10^{-13}\end{array}$ & $\begin{array}{l}\text { RANDOM UNCERTAINTY } \\
\text { IN } 10^{-13}\end{array}$ & $\begin{array}{c}\text { SYSTEMATIC UNCERTAINTY } \\
\text { IN } 10^{-13}\end{array}$ \\
\hline NBS & NBS 3 & $40330-40390$ & 11.8 & 5.2 & 2.5 \\
\hline NRC & NRC CS3 & $40221-40587$ & 3.2 & 13.3 & 7.0 \\
\hline NRC & NRC CS3 & 40587-40709 & 5.4 & 13.3 & 7.0 \\
\hline NRC & NRC CS3 & $40709-40952$ & 9.9 & 13.3 & 7.0 \\
\hline NRC & NRC CS3 & $40952-41072$ & 1.4 & 13.3 & 7.0 \\
\hline NRC & NRC CS3 & $41072-41139$ & 4.1 & 13.3 & 7.0 \\
\hline PTB & РTB CS1 & 40494-40644 & 16.6 & 1.0 & 1.8 \\
\hline PTB & PTB CS1 & 40749-40809 & 14.9 & 1.9 & 1.8 \\
\hline PTB & PTB CS1 & 40889-40949 & 13.9 & 2.0 & 1.8 \\
\hline PTB & PTB CS 1 & 41449-41509 & 12.8 & 0.7 & 1.8 \\
\hline PTB & PTB CS1 & $41729-41789$ & 11.7 & 1.2 & 1.8 \\
\hline PTB & PTB CS 1 & $42249-42309$ & 8.5 & 1.6 & 1.8 \\
\hline PTB & РTB CS 1 & $42365-42425$ & 11.2 & 1.7 & 1.8 \\
\hline PTB & PTB CS1 & $42429-42489$ & 11.6 & 1.8 & 1.8 \\
\hline PTB & РTB CS 1 & $42585-42645$ & 9.2 & 1.4 & 1.8 \\
\hline PTB & PTB CS1 & $42629-42689$ & 11.8 & 1.8 & 1.8 \\
\hline РTB & РTB CS 1 & $42749-42809$ & 11.1 & 1.6 & 1.8 \\
\hline NBS & NBS 5 & $41681-41741$ & 12.8 & 2.3 & 2.7 \\
\hline NBS & NBS 5 & $41696-41756$ & 11.2 & 2.5 & 2.7 \\
\hline NBS & NBS 5 & $41731-41791$ & 11.2 & 5.2 & 2.7 \\
\hline NBS & NBS 5 & $41747-41807$ & 12.5 & 2.8 & 2.7 \\
\hline NBS & NBS 5 & $41934-41994$ & 8.7 & 2.4 & 2.7 \\
\hline NBS & NBS 4 & 41896-41956 & 4.3 & 5.2 & 2.5 \\
\hline NBS & NBS 4 & $42019-42079$ & 11.1 & 3.1 & 0.5 \\
\hline NBS & NBS 4 & $42056-42116$ & 11.7 & 3.1 & 0.5 \\
\hline
\end{tabular}

Data used for evaluating the duration of the tai second. Gravitational frequency corrections are applied. The frequencies are expressed at sea level.

In terms of number, several tens of fountains have been, and are being, built and operated in more than ten laboratories participating to UTC. A number of Cs fountains are operated as PFS and are used to ensure the accuracy of UTC/TAI through the steering of TAI. One $\mathrm{Rb}$ fountain is also operated as a secondary frequency standard and similarly used in TAI steering.

In terms of uncertainty budget, the estimation of all systematic frequency shifts was around $1 \times 10^{-15}$ in the best cases in the early 2000s and decreased to around $2 \times 10^{-16}$ in the best cases in the 2010s. The fractional frequency stability of the fountains has also been improved by about one order of magnitude over the period.

In terms of robustness, primary standard fountains are now often operated with a duty time in excess of $99 \%$, and have generally been above $90 \%$ for most of their history of reports to TAI.

The presentation will review the frequency standards currently provided to the BIPM, and the resulting accuracy of TAI and of TT(BIPM).

\subsection{Accuracy of TAI and TT(BIPM)}

Primary standards have been used to estimate the rate of TAI, or the duration of the scale unit of TAI with respect to the SI second, noted $d$, since its inception. Each month with the TAI computation, the BIPM publishes (Section 3 of Circular T) the estimation of $d$ by individual PSFS measurements and its uncertainty. This value $d$ is the opposite of the fractional frequency deviation of TAI from its ideal definition, that is, $d=y$ (TT-TAI) or $d=\mathrm{y}(\mathrm{Cs}-\mathrm{TAI})$. Besides TAI, the BIPM generates every year another post-processed timescale, TT(BIPMyy), produced beginning of year [20yy +1$] \cdot{ }^{[34]}$ Like TAI, TT(BIPM) gets its accuracy from PFS and, since 2012, also from secondary standards. To put it simply TT(BIPM) can be considered as a moving average of the available PSFS. The monthly $d$ value and its uncertainty as well as TT(BIPM) and its estimated accuracy are computed with a specific algorithm described in ref. [35] and the computed uncertainties directly depend on the uncertainties reported for the PSFS.

Over the last two decades the number of high accuracy Cs fountains contributing to TAI has increased: from only two over the period 2000-2002, their number has reached eleven over the period 2010-2011 and is recently (2016-2018) at about 8-9. In addition, PFS evaluations have been carried out on a more regular basis, so that the number of individual measurements of the TAI frequency per year has increased about tenfold over the period. Finally the uncertainty assigned to each evaluation has decreased from the low $10^{-15}$ to the low $10^{-16}$ in the best cases.

The evolution of the characteristics of PFS can also be visualized through Tables 1-3 which provide, respectively: 
Table 2. Typical characteristics of the primary standards reported in 1996 (Data taken from Table 6 of ref. [36]). One can note the first appearance of a Cs fountain LPTF FO1.

\begin{tabular}{|c|c|c|c|c|}
\hline Standard & Unc. $(1 \sigma)$ & Operation & Comparison with & Transfer to TAI \\
\hline CRL Cs 1 & $1.1 \times 10^{-13}$ & Discontinuous & UTC(CRL) & $60 \mathrm{~d}$ \\
\hline LPTF JPO & $1.1 \times 10^{-13}$ & Discontinuous & UTC(OP) & $10 d$ \\
\hline LPTF FO1 & $0.3 \times 10^{-14}$ & Discontinuous & $\mathrm{H}$ maser & $5 \mathrm{~d}$ or $10 \mathrm{~d}$ \\
\hline NIST NIST-7 & $1 \times 10^{-14}$ & Discontinuous & $\mathrm{H}$ maser & $5 \mathrm{~d}$ or $10 \mathrm{~d}$ \\
\hline NRC CsV & $\sim 1 \times 10^{-13}$ & Continuous & TAI & $60 d$ \\
\hline NRC CsVI A & $\sim 1 \times 10^{-13}$ & Continuous & TAI & $60 d$ \\
\hline NRC CsVIC & $\sim 1 \times 10^{-13}$ & Continuous & TAI & $60 d$ \\
\hline РTB CS 1 & $3 \times 10^{-14}$ & Continuous & TAI & $60 d$ \\
\hline PTB CS2 & $1.5 \times 10^{-14}$ & Continuous & TAI & $60 d$ \\
\hline PTB CS3 & $1.4 \times 10^{-14}$ & Continuous & TAI & $60 d$ \\
\hline SU MCsR 102 & $5 \times 10^{-14}$ & Discontinuous & UTC(SU) & $60 d$ \\
\hline
\end{tabular}

Table 3. Typical characteristics of the primary and secondary standards reported in 2017 (Data taken from Table 6 of ref. [37]).

\begin{tabular}{llccc}
\hline Primary standard & Type / selection & Type B std. uncertainty/ $10^{-15}$ & Comparison with & Number/typical duration of comp. \\
\hline IT-CSF2 & Fountain & 0.17 & H maser & $3 / 20 \mathrm{~d}$ to $30 \mathrm{~d}$ \\
NIM5 & Fountain & 1.4, then 0.9 & $\mathrm{H}$ maser & $3 / 15 \mathrm{~d}$ to $20 \mathrm{~d}$ \\
PTB-CS1 & Beam /Mag. & 8 & TAl & $12 / 25 \mathrm{~d}$ to $35 \mathrm{~d}$ \\
PTB-CS2 & Beam /Mag. & 12 & TAl & $12 / 25 \mathrm{~d}$ to $35 \mathrm{~d}$ \\
PTB-CSF1 & Fountain & 0.35 to 0.40 & H maser & $7 / 15 \mathrm{~d}$ to $30 \mathrm{~d}$ \\
PTB-CSF2 & Fountain & 0.20 to 0.24 & H maser & $12 / 20 \mathrm{~d}$ to $35 \mathrm{~d}$ \\
SU-CSFO2 & Fountain & 0.24 & H maser & $6 / 15 \mathrm{~d}$ to $35 \mathrm{~d}$ \\
SYRTE-FO2 & Fountain & 0.24 to 0.37 & H maser & $9 / 10 \mathrm{~d}$ to $35 \mathrm{~d}$ \\
Secondary standard & Type & Type B std. uncertainty/ 10-15 & Comparison with & Number/typical duration of comp. \\
\hline SYRTE-FORb & Fountain & 0.28 to 0.30 & H maser & $9 / 10 \mathrm{~d}$ to $35 \mathrm{~d}$ \\
SYRTE-Sr2 & Lattice & 0.04 or 0.20 & H maser & $4 / 10 \mathrm{~d}$ to $20 \mathrm{~d}$ \\
SYRTE-SrB & Lattice & 0.05 & H maser & $1 / 15 \mathrm{~d}$ \\
\hline
\end{tabular}

- The list of frequency standards contributing to TAI over 1975 and the previous years, with a relative accuracy in the low $10^{-13}$. Note that Table 1 covers about 6 years and the number of reported measurements and of reporting laboratories is small compared to the following decades.

- The typical characteristics of the primary standards reported in 1996. One can note the first appearance of a Cs fountain, LPTF FO1, with a relative accuracy of $3 \times 10^{-15}$ while other beam devices now attain the low $10^{-14}$.

- The typical characteristics of the primary and secondary standards reported in 2017. One can note an uncertainty as low as $2 \times 10^{-16}$ and the introduction of lattice clocks (SFS) in which cold atoms are trapped by counter-propagating laser beams.

A statistical study comparing the reports of nine fountains over the period 2006-2012 showed no significant discrepancy in the evaluations reported to the BIPM. ${ }^{[38]}$ Comparisons to TT(BIPM) allowed determining an average frequency bias for each fountain and showed that the distribution of these biases is well consistent with the stated accuracies of the fountains, although some of the biases are statistically significant. Validation of the stated uncer- tainties of individual fountains allows validation of the uncertainties obtained for the monthly estimation of the TAI frequency and for the relative frequency accuracy of TT(BIPM), which have been at the level of $2 \times 10^{-16}$ or below since 2012 (see Figure 2). It is interesting to note that the development of fountains has marked the period shown on Figure 2, with the dip around 1996 due to a few evaluations of the first fountain LPTF FO1 (see Table 2) and the notable improvement since year 2000 due to the progressive introduction of more, and more accurate, fountains. However, the improvement was not continuous. Months without fountain measurements were not rare in the 2000s as attested by the variations in the uncertainty of TT(BIPM); furthermore, the improvement has slowed since $\approx 2012$ because clock uncertainties have become equivalent to or smaller than the frequency transfer uncertainty which is not improving. Note that the uncertainty in the monthly evaluation of the TAI frequency is slightly larger than the corresponding monthly value in the accuracy of TT(BIPM) because the former is computed with PSFS evaluations available at the time of the monthly computation while the latter is computed in deferred time so that more PSFS evaluations are available (those for later months or submitted with delay). 


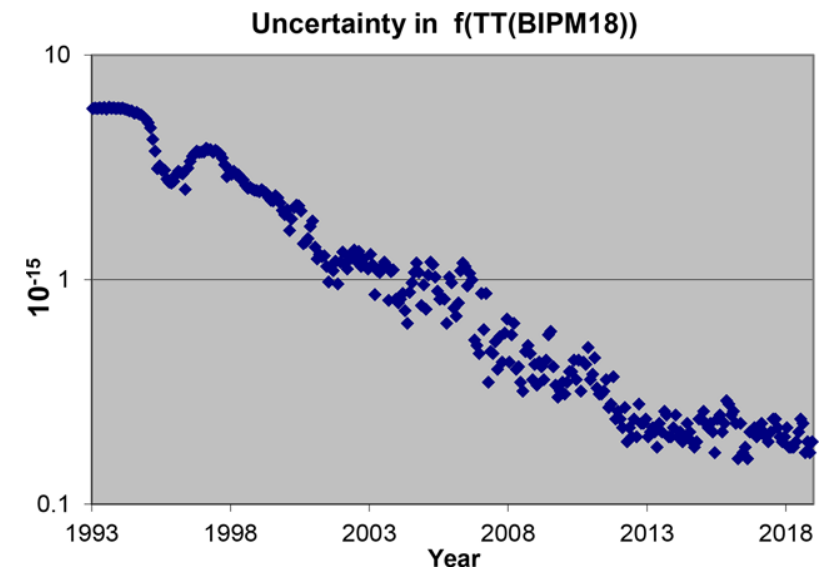

Figure 2. Monthly estimation of the relative frequency uncertainty of TT(BIPM 18), representing its accuracy.

\subsubsection{Fountain-Driven Timescales}

With the improvement in stability, reliability, and robustness of the fountain clocks it became possible to operate them without interruption for extended periods of time, opening the way to their use in continuous timescales. This became a reality over the year 2010, both with fountains designed to work as continuously running clocks or with primary standard fountains.

In the first category, the USNO started end 2011 reporting four $\mathrm{Rb}$ fountains as clocks for participation to the UTC ensemble. These $\mathrm{Rb}$ fountains have a typical instability of order $1 \times 10^{-15}$ at one day averaging and show white frequency noise behavior for extended periods. ${ }^{[39]}$ They were first weighted in the UTC ensemble in May 2012 and have contributed with the maximum weight every month since that time, typically providing $4 \%$ of TAI. Other laboratories follow this route (VNIIFTRI in the Russian Federation, AOS-GUM in Poland) and their effective participation to UTC is expected soon. Fountains in this category are mostly Rb fountains, an atom for which the laser sources are more reliable and efficient. Eventually, if this category of clocks becomes numerous enough and used in many laboratories, it could be advantageous to modify the UTC weighting scheme so that such clocks with superior long-term stability and predictability can gain a larger weight. Indeed, the weight of a clock in the ensemble, while derived from the clock's predictability, ${ }^{[40]}$ is presently limited to $4 / N$ where $N$ is the number of clocks considered for weighting. The maximum weight is designed to safeguard the scale from being dominated by a small number of clocks but it may also prevent the scale from benefiting of the best clocks. The maximum weight has been at about $1 \%$ since 2014 but the cumulated weight of all clocks at maximum weight is increasing, a sign that the maximum weight may not be optimal. ${ }^{[4]}$ Finally it should be mentioned that, besides atomic fountains, other clock designs using cold atoms are becoming available, some on a commercial basis, ${ }^{[42]}$ and should also contribute to the UTC ensemble.

In the second category, a few laboratories participating to UTC explicitly steer their UTC(k) using fountains. The PTB was the first laboratory to do so since February $2010,{ }^{[43]}$ where the Cs primary fountain is used both for its short-term stability and as a reference to extrapolate the rate of UTC(PTB) with respect to TAI.
The LNE-SYRTE has also implemented such a steering since October 2012. ${ }^{[4]}$ In both cases the difference [UTC-UTC $\left.(k)\right]$ has remained within an interval of total amplitude $14 \mathrm{~ns}$ at all times since the implementation, both series showing a standard deviation of 2.4 ns over close to 9 years for the PTB and close to 7 years for the LNE-SYRTE. This level of performance in the realization of UTC over very long periods has also been achieved by USNO and has been matched by a few other laboratories in recent years. Note also that the BIPM provides UTCr, a weekly prediction of $\mathrm{UTC}^{[45]}$ which differs from UTC by less than $2 \mathrm{~ns}$ in absolute value since July 2017, so that the work needed to generate a real-time realization of UTC by laboratories may be simpler. The performance in such realization of UTC is ultimately limited by the instability of UTC itself and by that of the time transfer link of the laboratory.

With the availability of several fountains running continuously (fountain clocks) or nearly continuously (primary and secondary standards) it can be envisioned to use them to generate an ensemble timescale in a similar way as it is done for TAI. This has been attempted in $2013^{[46]}$ with a repeat in 2015. It was shown that, besides the four continuously running USNO fountains, primary and secondary standards could provide an additional three to four clocks if gaps up to 10 days are allowed to be interpolated. The performance of such an "International Fountain Time" has been evaluated to be similar to TAI or TT(BIPM), around $3 \times 10^{-16}$ at one month averaging. However, even now, such a timescale would be based on too few clocks and would not be robust and reliable enough to be considered independently. It is worth mentioning that the frequency transfer noise of the TAI links (typically $2-3 \times 10^{-16}$ ) is a limiting factor when considering clocks in a few remote laboratories. However, such a limitation might be waived in post-processing as the few needed links could be computed with the IPPP (precise point positioning with integer ambiguity resolution) technique ${ }^{[47]}$ yielding frequency transfer uncertainty well below $1 \times 10^{-16}$.

\section{Present Situation}

The cesium fountains realize today the SI second with uncertainties of about $1-2 \times 10^{-16}$, and no significant improvement is expected beyond this limit. About a dozen of these artifacts operated in major metrology institutes contribute frequency measurements which, integrated in an algorithm developed at the BIPM confer accuracy to the reference atomic timescale. The comparison of these standards and their inclusion in TAI is possible with the current techniques of frequency transfer, based on mono and bi-directional comparisons via satellites and optical fiber connections.

Although a timescale based only on Cs fountains does not seem presently viable, they are used to improve the accuracy of some of the local atomic timescales $\operatorname{UTC}(k)$.

In 2001 the CCTF recognized that there were new atoms and ions being studied as potential optical frequency standards that could allow the use of optical transitions as practical frequency standards offering direct microwave outputs from such standards. One of these standards could provide the basis for a future definition of the second, and the CCTF focused on the desirability of reviewing accurate frequency measurements of such atom and 


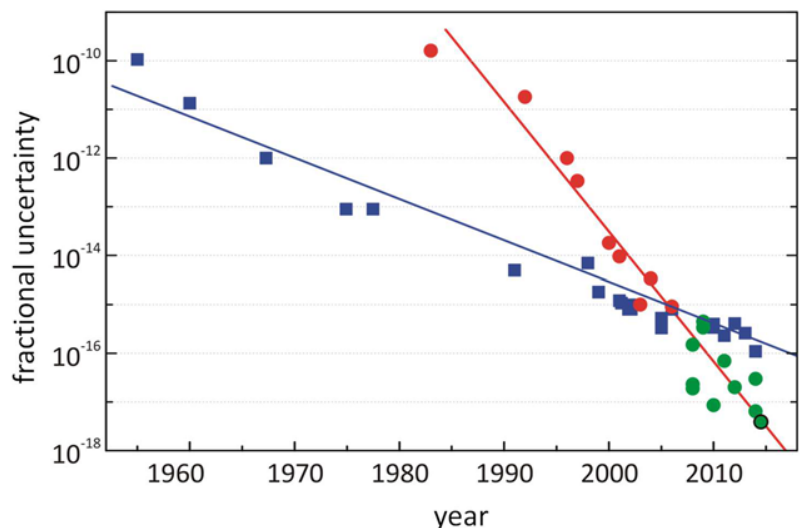

Figure 3. Evolution of the fractional uncertainty to realize the unperturbed line center of primary atomic cesium clocks (squares) and of optical frequency standards (dots). Red dots show the fractional uncertainties of optical frequency standards directly related to the cesium atomic clock, green dots refer to published estimated standard uncertainties to realize the unperturbed line center. Reproduced with permission. ${ }^{[48]}$ Copyright 2017, IOP.

ion transition frequencies made relative to the cesium frequency standard. In addition, the femtosecond optical frequency comb offered solutions to the longstanding problem of a convenient and accurate clockwork that linked the optical and microwave regions and allowed for frequency comparisons between optical frequency standards with very different frequencies. To better organize the work, a list of radiations suitable as Secondary Representations of the Second (SRS) has been established by the CIPM and is updated whenever the CCTF decides that relevant new information is to be included ${ }^{[49]}$ As of 2017, the list contains nine transitions, eight in the optical region and one in the microwaves. Besides the frequency measurements compared against cesium, a number of frequency ratios have been determined between optical atomic clocks with much smaller uncertainties that those obtained with cesium or any other microwave clock. A description of the list of frequencies has been published in ref. [48].

\section{Conclusion}

The atomic definition of the SI unit of time was adopted in 1967. Since then, the cesium frequency standards have provided the realization of the second with increasing accuracy. The astronomical seconds never experienced a comparable progress.

Also, cooperative work was necessary among institutes maintaining standards and local timescales to assure their comparability and dissemination. International organizations and scientific unions played a fundamental role in establishing the basis for this coordination.

The achievement of new standards, with uncertainties of order $10^{-18}$ challenges the metrology community with the possibility of a future redefinition of the second. Figure 3 schematizes the evolution of the fractional uncertainty of the determination of the cesium transition since Essen's clock, together with that of the optical standards.

This new optical frequency metrology is opening new fields and applications; with the achieved accuracy and stability of the optical atomic clocks it is possible to determine the differences in the gravitational potential of two locations on the Earth; optical clocks can help to test fundamental theories and possible variations of the fundamental constants.

In view of the present status of the frequency standards development and of the brilliant perspectives of progress they could bring to the physical sciences, there seems to be an adequate momentum for envisaging a redefinition of the second in the next decade.

\section{Acknowledgements}

This article is part of the Special Issue on The Revised SI: Fundamental Constants, Basic Physics and Units, highlighting the revision and redefinition of the International System of Units (SI) to come into effect in May 2019.

\section{Conflict of Interest}

The authors declare no conflict of interest.

\section{Keywords}

cesium, primary frequency standard, SI second

Received: February 18, 2019

Revised: March 18, 2019

Published online: April 14, 2019

[1] L. Essen, J. V. L. Parry, Nature 1955, 176, 280.

[2] A. Scheibe, U. Adelsberger, Phys. Z. 1936, 37, 185.

[3] Transactions of the International Astronomical Union, Proc.8th General Assembly, Rome 1952 (Ed: P.T. Oosterhoff), vol. VIII Cambridge University Press, New York 1954.

[4] S. Newcomb, Astronomical Papers Prepared for the Use of the Annual Ephemeris and Nautical Almanac, Vol VI, Part I: Tables of the Sun, US Govt. Printing Officer, Washington DC 1895, 9.

[5] Bureau International des Poids et Mesures, Comptes rendus de la 11 ème Conférence Générale des Poids et Mesures (1960) 1961, 86.

[6] I. I. Rabi, Phys. Rev. 1937, 51, 652.

[7] I. I. Rabi, J. R. Zacharias, S. Millman, P. Kusch, Phys. Rev. 1938, 53, 318.

[8] N. F. Ramsey, Phys. Rev. 1950, 78, 695.

[9] S. Leschiutta, Metrologia 2005, 42, S10.

[10] W. Markowitz, R. G. Hall, L. Essen, J. V. L. Parry, Phys. Rev. Lett. 1958, 1, 105.

[11] Bureau International des Poids et Mesures, Metrologia 1968, 4, 41.

[12] L. Essen, J. V. L. Parry, Phyl. Trans. of the Royal Soc. of London Series A, Mathematical and Physical Sciences 1957, 250, 45.

[13] B. Guinot, E. F. Arias, Metrologia 2005, 42, S20.

[14] Transactions of the VIII International Astronomical Union General Assembly, 1952.

[15] Transactions of the IX International Astronomical Union General Assembly 1955.

[16] Resolution 1, Procès verbaux des séances du CIPM, p. 77, 1956.

[17] Recommendation ITU-R, TF.460-6, Standard frequencies and time signal emissions, www.itu.int/rec/R-REC-TF.460-6-200202-1/en (accessed: April 2019).

[18] L. Cutler, Metrologia 2005, 42, S90.

[19] Bureau International des Poids et Mesures, Metrologia 1972, 8, 32. 
[20] Bureau International des Poids et Mesures, Metrologia 1975, 11, 179.

[21] BIPM Circular T, monthly, https://www.bipm.org/en/bipm-services/ timescales/time-ftp/Circular-T.html (accessed: April 2019).

[22] Bureau International des Poids et Mesures, Resolution 2 of the 26th General Conference on Weights and Measures (2018), www.bipm.org/en/CGPM/db/26/2/ (accessed: April 2019).

[23] G. Petit, in Proc. 7th Symposium on frequency standards and metrology, Ed: L. Maleki, World Scientific 2009, 475.

[24] J. Vanier, C. Audoin, Metrologia 2005, 42, S31.

[25] N. F. Ramsey, Metrologia 2005, 42, S1.

[26] J. R. Zacharias, J. G. Yates, R. D. Haun, Proc. IRE 1955, 43, 364

[27] A. Clairon, C. Salomon, S. Guellati-Khelifa, W. Phillips, Europhys. Lett. 1991, 16, 165

[28] R. Wynands, S. Weyers, Metrologia 2005, 42, S64.

[29] T. P. Heavner, E. A. Donley, F. Levi, G. Costanzo, T. E. Parker, J. H. Shirley, N. Ashby, S. Barlow, S. R. Jefferts, Metrologia 2014, 51, 174.

[30] F. Levi, D. Calonico, C. E. Calosso, A. Godone, S. Micalizio, G. Costanzo, Metrologia 2014, 51, 270.

[31] G. J. Dick, Proc. 19th Ann. PTTI Meeting 1987, 133.

[32] A. Jallageas, L. Devenoges, M. Petersen, J. Morel, L. G. Bernier, D. Schenker, P. Thomann, T. Südmeyer, Metrologia 2018, 55, 366.

[33] Bureau international de l'heure, Annual Report for 1975, https:// www.bipm.org/en/bipm-services/timescales/time-ftp/annual-reports. html (accessed: April 2019).

[34] TT(BIPM), ftp://ftp2.bipm.org/pub/tai/ttbipm/.

[35] J. Azoubib, M. Granveaud, B. Guinot, Metrologia 1977, 13, 87.

[36] Bureau international des poids et mesures, Anual Report of the
Time Section for 1996, https://www.bipm.org/en/bipm-services/ timescales/time-ftp/annual-reports.html (accessed: April 2019).

[37] Bureau international des poids et mesures, Anual Report on Time Activities for 2017, https://www.bipm.org/en/bipm-services/ timescales/time-ftp/annual-reports.html (accessed: April 2019).

[38] G. Petit, G. Panfilo, IEEE Trans. Instrum. Meas. 2013, 62, 1550.

[39] S. Peil, J. L. Hanssen, T. B. Swanson, J. Taylor, C. R. Ekstrom. Metrologia 2014, 51, 263.

[40] G. Panfilo, A. Harmegnies, Proc IFCS-EFTF 2013, 2013, 652.

[41] G. Petit, Metrologia 2003, 40, S252.

[42] Commercially available atomic clock based on cold atoms, https://www.muquans.com/index.php/products/mclock (accessed: April 2019).

[43] A. Bauch, S. Weyers, D. Piester, E. Staliuniene, W. Yang, Metrologia 2012, 49, 180.

[44] G. D. Rovera, S. Bize, B. Chupin, J. Guéna, Ph. Laurent, P. Rosenbusch, P. Uhrich, M. Abgrall, Metrologia 2016, 53, S81.

[45] G. Petit, E. F. Arias, A. Harmegnies, G. Panfilo, L. Tisserand, Metrologia 2014, 51, 33.

[46] Petit, G., in Proceedings of the 45th Annual Precise Time and Time Interval Systems and Applications Meeting, Bellevue, Washington, December 2013, 2014, 265.

[47] G. Petit, A. Kanj, S. Loyer, J. Delporte, F. Mercier, F. Perosanz, Metrologia 2015, 52, 301.

[48] F. Riehle, P. Gill, F. Arias, L. Robertsson, Metrologia 2018, 55, 188.

[49] List of recommended frequencies for secondary representations of the second, https://www.bipm.org/en/publications/mises-enpratique/standard-frequencies.html (accessed: April 2019). 